\title{
PHOTOTRANSISTOR-BASED OPTOELECTRONIC TWEEZERS FOR CELL MANIPULATION IN HIGHLY CONDUCTIVE SOLUTION
}

\author{
H. Y. Hsu, A. T. Ohta, P. Y. Chiou, A. Jamshidi, and M. C. Wu \\ Berkeley Sensor and Actuator Center and Dept. of Elec. Engineering and Computer Sciences \\ Berkeley, California, USA \\ Tel: 1-510-642-1023; Fax: 1-510-643-5817; email: hsu@eecs.berkeley.edu
}

\begin{abstract}
We report on a novel phototransistor-based optoelectronic tweezers (OET) that, for the first time, enables operation in high-conductivity solutions, such as physiological buffers and cell culture media. This new capability allows the manipulation of cells in biocompatible environments. The controlled transport of $\mathrm{HeLa}$ and Jurkat cells at velocities of up to $35 \mu \mathrm{m} / \mathrm{s}$ in phosphate-buffered saline (PBS) solution has been demonstrated.
\end{abstract}

Keywords: Optoelectronic tweezers, cell manipulation, dielectrophoresis

\section{INTRODUCTION}

Conventional noninvasive cell manipulation techniques include optical tweezers [1] and electrode-based dielectrophoresis (DEP) [2]. Optical tweezers offer high resolution and flexibility, but have a limited manipulation area owing to tight focusing requirements, limiting throughput of cellular-scale objects. In contrast, DEP provides high throughput, but lacks the flexibility of optical control for controlling specific individual particles. Optoelectronic tweezers (OET) [3] utilizes optically-induced dielectrophoresis to manipulate cells and other micro- and nano-particles, allowing OET to retain the flexibility of optical control, while offering increased parallel manipulation capabilities as compared to optical tweezers.

The OET manipulation of red and white blood cells and HeLa cells $[4,5]$ have been previously demonstrated, as well as the selective concentration of live human B cells from dead cells [3]. However, until now, OET has been limited by the usage of amorphous silicon as the photoconductive material of the device. The maximum photoconductivity of amorphous silicon (a-Si) allows OET to operate only in low-conductivity solutions (less than 0.1 $\mathrm{S} / \mathrm{m}$ ). Thus, to manipulate cells in a conventional OET device, the salts that are usually present in cell culture media are replaced by osmoticallyequivalent amounts of non-electrolytes to maintain the osmotic pressure on the cell membranes. These low-conductivity media are non-physiological, and eventually reduce cell viability [6]. The usage of non-physiological media also limits many biological applications, such as cell culturing and electroporation. Here, we report a novel phototransistor-based OET (Ph-OET) device that enables the manipulation of cells in highlyconductive physiological buffers and cell culture media.

\section{PRINCIPLE OF OPTOELECTRONIC TWEEZERS}

Figure 1 shows a schematic illustrating the principle of OET. A typical OET device consists of sandwiched layers consisting of a transparent indium-tin-oxide (ITO) electrode, liquid media, a photoconductive layer, and a bottom electrode. An $\mathrm{AC}$ voltage is applied between the top and bottom electrodes. To actuate the OET device, an optical pattern is projected onto the photoconductive layer. In the absence of light, the impedance of the photoconductive layer is higher than the impedance of the liquid media; thus, the majority of applied voltage is dropped across the photoconductor. In the presence of light, the impedance of the photoconductive layer is reduced so that the majority of voltage is dropped across the liquid layer in the illuminated area. This creates a non- 
uniform electric field profile. The particles experience a DEP force resulting from the interaction between the electric field and the induced electric dipole of the particles. In highlyconductive solutions, the cells experience negative DEP forces, which direct the cells away from the light pattern.

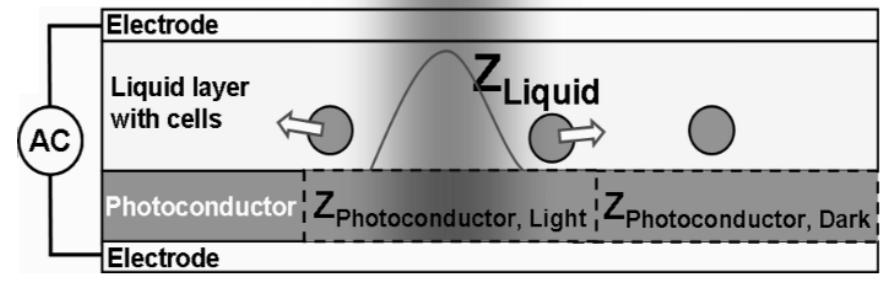

Fig. 1 Schematic illustrating the principle of optoelectronic tweezers. The optical illumination changes the local impedance of photoconductor layer, and creates an electric field gradient in liquid media, generating dielectrophoretic force.

To generate DEP force with the OET device, the impedance of the illuminated photoconductor must be lower than that of the liquid media. Previous OET devices utilize a-Si, which has a photoconductivity of approximately $0.01 \mathrm{~S} / \mathrm{m}$ under a light intensity of $100 \mathrm{~W} / \mathrm{cm}^{2}$. This low photoconductivity presents a great challenge for operation in highly-conductive solutions. To circumvent this limit, we have developed a novel Ph-OET device.

\section{PHOTOTRANSISTOR-BASED OET}

The schematic of the Ph-OET is shown in Figure 2. Similar to previous OET devices, the solution containing cells is sandwiched between an ITOcoated glass and a photoconductive layer. Here the photoconductive layer is comprised of pixelated single-crystalline silicon bipolar phototransistors. The structure of the phototransistors is shown in the inset of Figure 2. It consists of a heavily n-doped emitter $\left(\mathrm{N}=10^{20} \mathrm{~cm}^{-3}\right)$, a $\mathrm{p}$-doped base $\left(\mathrm{P}=10^{16} \mathrm{~cm}^{-}\right.$ $\left.{ }^{3}\right)$, and a lightly n-doped collector $\left(\mathrm{N}=6 \cdot 10^{14} \mathrm{~cm}^{-3}\right)$. The doping concentration is estimated with the processing simulation tool Tsuprem4, based on the actual process parameters. The Ph-OET is fabricated on a highly-doped substrate with a $5-\mu \mathrm{m}-$ thick, epitaxially-grown $\mathrm{Si}$ layer with light ndoping. The fabrication process is shown in Figure 3 . The phototransistor is patterned into square pixels of $10 \mu \mathrm{m} \times 10 \mu \mathrm{m}$, formed by deep-reactive-ionetching through the base. The $2-\mu \mathrm{m}$-wide trenches are filled with photoresist to avoid direct contact between the liquid and the base area. The light pattern is projected from the top through the ITOcoated glass.

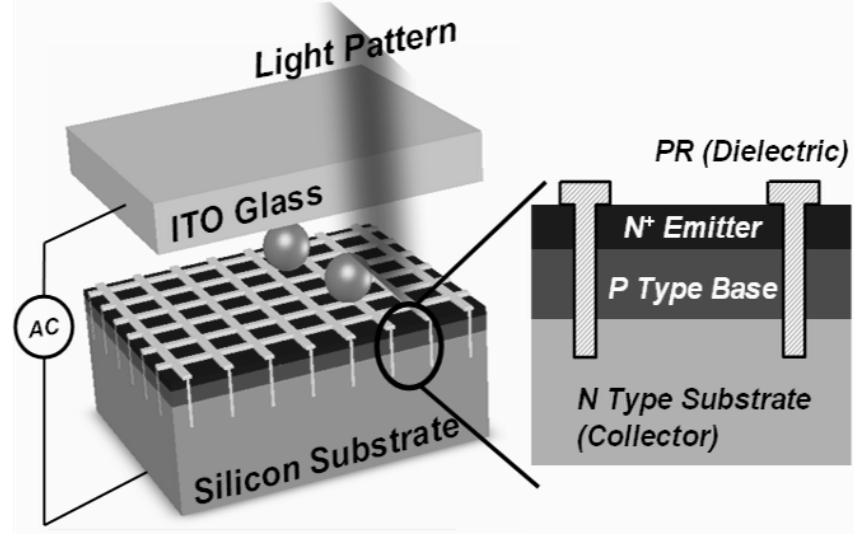

Fig. 2 Schematic of phototransistor-based OET device. The inset shows the phototransistor structure.

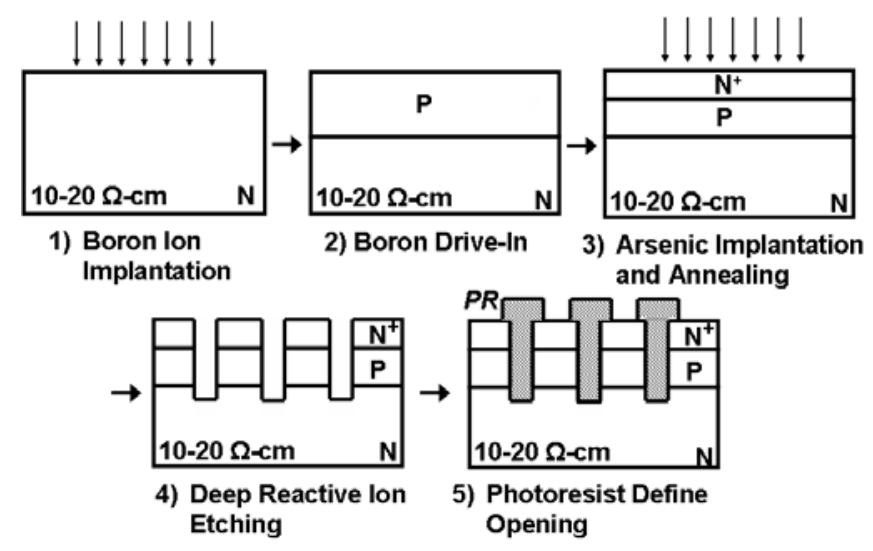

Fig. 3 Fabrication process of Ph-OET device.

The phototransistors offer much higher photoconductivity than the a-Si used in conventional OET, thanks to higher carrier mobility in single-crystalline silicon, and the large current gain of the phototransistor structure. Figure 4 shows 
the measured photoconductivity per unit area for the phototransistor structure and for a-Si. The phototransistor devices exhibited a photoconductivity that is more than 2 orders of magnitude higher than that of a-Si at an optical power density of $1 \mathrm{~W} / \mathrm{cm}^{2}$. To operate OET in physiological solutions with a conductivity of 1.5 $\mathrm{S} / \mathrm{m}$, the photoconductivity should be greater than $1.5 \mathrm{~S} / \mathrm{cm}^{2}$, assuming a liquid layer thickness of 100 $\mu \mathrm{m}$. Using the phototransistor device, the photoconductivity requirement is satisfied at a light intensity of less than $0.3 \mathrm{~W} / \mathrm{cm}^{2}$, while amorphous silicon requires a light intensity of higher than $50 \mathrm{~W} / \mathrm{cm}^{2}$. Thus, Ph-OET significantly reduces the optical power needed to operate in highconductivity solutions. The low optical power requirement reduces heating and avoids photodamage of biological samples [7].

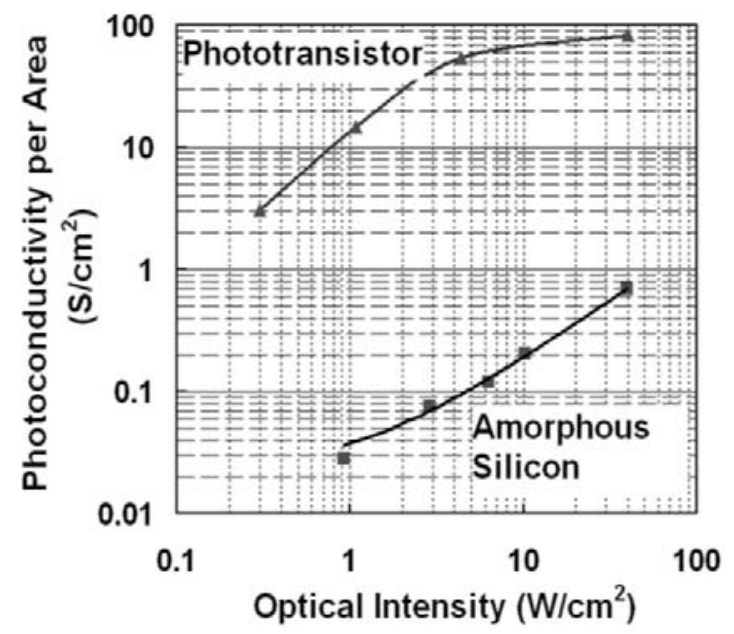

Fig. 4 Photoconductivity measurement comparison between the phototransistor and amorphous silicon.

\section{CELL MANIPULATION RESULTS}

We have successfully manipulated HeLa and Jurkat cells in phosphate-buffered saline solution (PBS) and Dulbecco's Modified Eagle Medium (DMEM) using the Ph-OET. Both solutions have a conductivity of $1.5 \mathrm{~S} / \mathrm{m}$. The experimental setup is shown in Figure 5. All experiments were done by applying a sinusoidal AC bias at a frequency of 9 MHz. Optical patterns were generated with a 658$\mathrm{nm}$ diode laser as the light source. A line pattern with an area of $250 \mu \mathrm{m} \times 50 \mu \mathrm{m}$ was projected onto the Ph-OET. When the optical line approaches the cells in the media, they experience a negative DEP force and are repelled away from the line pattern. Figure 6 shows three captured images of the cells as the optical line scans towards the right. Both HeLa cells experience repulsive forces in the highly conductive solutions, and were pushed from left to right by the scanning light pattern.

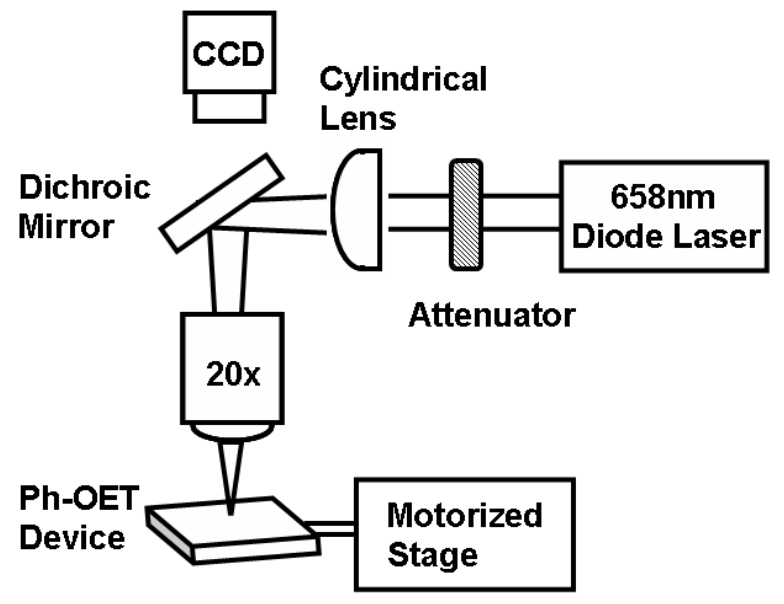

Fig. 5 Experimental setup for cell velocities measurement.

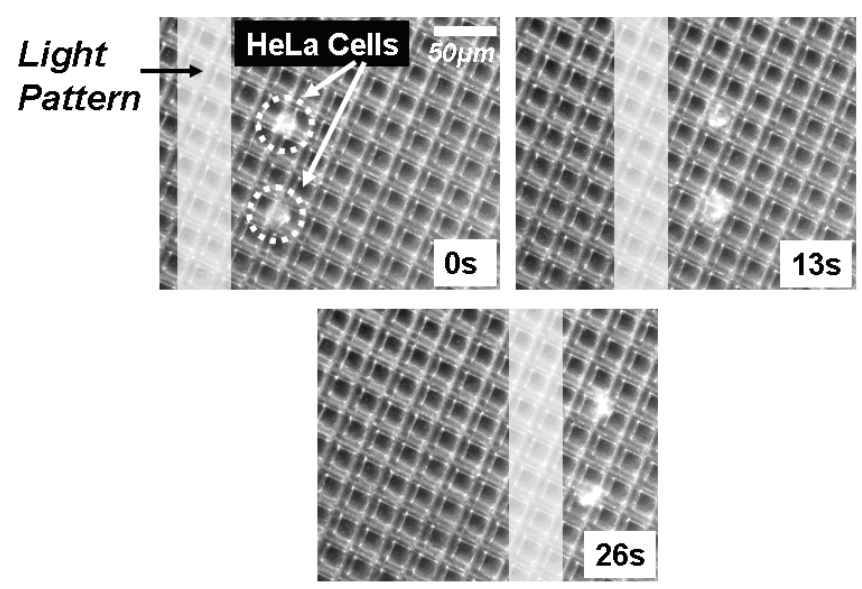

Fig. 6 Transport of two HeLa cells across field of view in PBS solution. The optical pattern is shaded for clarity.

To quantify cell velocity, the stage of the microscope is moved at a constant speed in relation to the optical pattern. The average cell manipulation velocity is defined by the stage speed in which the cells are able to keep up with the optical pattern. 
Cell manipulation velocities in PBS solution as a function of optical intensity and applied voltage are shown in Figure 7. The average cell velocities observed in PBS and DMEM are similar due to their identical conductivity.

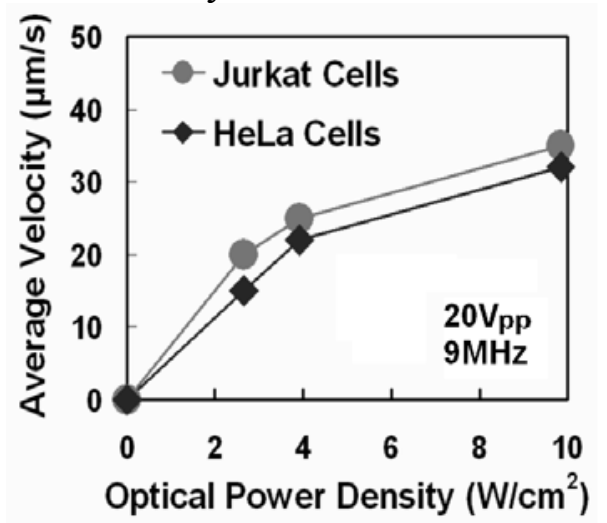

(a)

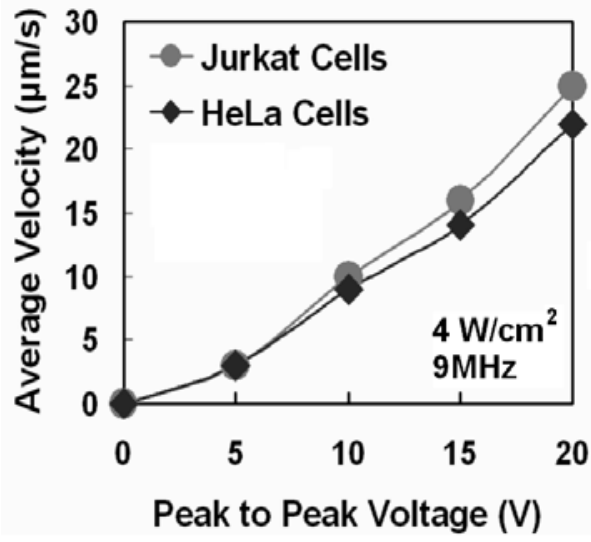

(b)

Fig. 7 Cell velocity measurement as a function of (a) optical power intensity and (b) applied voltage in PBS solution.

\section{CONCLUSION}

A novel phototransistor-based OET device has been developed for operations in highly-conductive solutions. The phototransistor has a photoconductivity that is more than 100 times greater than that of amorphous silicon, and thus requires a much lower optical intensity for OET operation. The manipulation of HeLa and Jurkat cells at velocities of up to $35 \mu \mathrm{m} / \mathrm{s}$ in PBS solution have been demonstrated. Furthermore, Jurkat cell movement at a velocity of $20 \mu \mathrm{m} / \mathrm{s}$ can be achieved with an optical power as low as $3 \mathrm{~W} / \mathrm{cm}^{2}$.

\section{ACKNOWLEDGMENT}

The project is supported by Applied Biosystems and the Institute for Cell Mimetic Space Exploration (CMISE), a NASA URETI. The author would like to thank the staff of the UC Berkeley Tissue Culture Facility for providing cells.

\section{REFERENCE}

[1] A. Ashkin, J. M. Dziedzic, J. E. Bjorkholm, and S. Chu, "Observation Of A Single-Beam Gradient Force Optical Trap For Dielectric Particles," Optics Letters, vol. 11, pp. 288-290, 1986.

[2] M. P. Hughes, "Strategies for dielectrophoretic separation in laboratory-on-a-chip systems," Electrophoresis, vol. 23, pp. 2569-2582, 2002.

[3] P. Y. Chiou, A. T. Ohta, and M. C. Wu, "Massively parallel manipulation of single cells and microparticles using optical images," Nature, vol. 436, pp. 370-372, 2005.

[4] A. T. Ohta, C. Pei-Yu, A. Jamshidi, H. Hsan-Yin, M. C. Wu, H. L. Phan, S. W. Sherwood, J. M. Yang, and A. N. K. Lau, "Spatial cell discrimination using optoelectronic tweezers," presented at 2006 Digest of the LEOS Summer Topical Meetings (IEEE Cat. No. 06TH8863C). IEEE. 2006, pp. 23-4. Piscataway, NJ, USA.

[5] P. Y. C. A. T. Ohta, T. H. Han, J. C. Liao, U. Bhardwaj, E. R. B. McCabe, F. Yu, R. Sun, M. C. $\mathrm{Wu}$, "Manipulation of live red and white blood cells via optoelectronic tweezers," presented at Proceeding of The International Conf. on Bio-NanoInformatics (BNI) Fusion, July 2005.

[6] J. Voldman, "Electrical forces for microscale cell manipulation," Annual Review Of Biomedical Engineering, vol. 8, pp. 425-454, 2006.

[7] K. C. Neuman, E. H. Chadd, G. F. Liou, K. Bergman, and S. M. Block, "Characterization of photodamage to Escherichia coli in optical traps," Biophysical Journal, vol. 77, pp. 2856-2863, 1999. 